\title{
Aplicación de la descarga luminiscente anormal como una fuente calórica
}

\author{
Applying anormal glow discharge as a caloric source
}

\author{
Iván Supelano García ${ }^{a *}$ \\ Armando Sarmiento Santos ${ }^{b}$ \\ Carlos Arturo Parra Vargas ${ }^{c}$
}

Recepción: 08 de junio de 2016

Aceptación: 21 de julio de 2017

\begin{abstract}
Resumen
La tecnología del plasma es una ciencia que desde sus comienzos se encuentra en una constante evolución, desde aplicaciones para el tratamiento de materiales hasta aplicaciones ambientales. En particular el plasma de la descarga luminiscente ofrece una amplia gama de aplicaciones desde aplicaciones en plasmo-química hasta tratamientos termoquímicos de materiales. En el presente trabajo se busca aprovechar la generación de calor por parte de la descarga luminiscente de baja presión en corriente continua, debido a la transferencia de energía cinética desde el plasma hacia los electrodos. Para ello se diseñó un reactor cilíndrico que genera una descarga luminiscente de baja presión en una atmósfera de argón. El cátodo de la descarga lo constituye el electrodo interno, mientras que el ánodo, y al mismo tiempo la pared de la cámara, lo constituye un cilindro metálico externo, concéntrico al cátodo. Para mantener la descarga se utilizó una fuente de corriente continua pulsada y la descarga se generó a varias corrientes y en diferentes atmósferas gaseosas $\left(\mathrm{N}_{2}, \mathrm{H}_{2}\right.$ y $A r$ ). Mediante medidas eléctricas de la descarga se determinó la potencia y consumo energético del reactor, comparándolo con los valores obtenidos para un dispositivo calefactor comercial basado en resistencias eléctricas.
\end{abstract}

Palabras clave: Descarga luminiscente, temperatura del cátodo, transferencia de calor.

\begin{abstract}
Plasma technology is a science that is in a constant evolution from its beginnings, from applications from materials treatment until environmental applications. Particularly the glow discharge plasma offers a wide variety of uses, including plasma-chemical applications and thermo-chemical materials treatment. In the present work seeks to exploit the heat generation by the low pressure glow discharge, which is due to kinetic energy transfer from the plasma toward the electrodes. For this purpose a cylindrical reactor that generates a low-pressure glow discharge in gas argon was designed. The discharge cathode constitutes the internal electrode, while the anode, which also constitutes the chamber wall, is a metallic cylinder concentric to the cathode. To sustain the glow discharge a source of pulsed direct current is used and the discharge was generated at several currents in different gas atmospheres $\left(\mathrm{N}_{2}, \mathrm{H}_{2}\right.$ y $\left.\mathrm{Ar}\right)$. Through electrical measurements of the discharge parameters, power and energy consumption of the reactor is determined. These are compared with the values obtained for a commercial heating device based on electrical resistance.
\end{abstract}

Keywords: Glow discharge, cathode temperature, heat transferring.

\footnotetext{
${ }^{\text {a }}$ Universidad Pedagógica y Tecnológica de Colombia, Grupo Superficies Electroquímica y Corrosión.

* Correo electrónico: ivan.supelano@uptc.edu.co

b Universidad Pedagógica y Tecnológica de Colombia, Grupo Superficies Electroquímica y Corrosión.

Correo electrónico: asarmiento.santos@uptc.edu.co

${ }^{\mathrm{c}}$ Universidad Pedagógica y Tecnológica de Colombia, Grupo Física de Materiales.

Correo electrónico: carlos.parra@uptc.edu.co
} 


\section{Introducción}

El plasma de descarga luminiscente generada en diferentes atmosferas y con diferentes diseños de reactores se ha usado en diferentes aplicaciones tecnológicas, por ejemplo, en procesos metalúrgicos como el tratamiento de superficies $[1,2]$, procesamiento de materiales $[3,4]$ y análisis de materiales sólidos (las técnicas más usadas en análisis directo de materiales solidos son la espectroscopia de masas y la descarga luminiscente con análisis de espectrometría de emisión óptica) [5]. Más recientemente la descarga luminiscente se ha utilizado en aplicaciones ambientales como la producción de hidrogeno y tratamiento de desechos [6].

En el caso de tratamiento de materiales se encuentra reportado el uso de la descarga luminiscente en atmósferas de aire [4], el principio se basa en que la región del cátodo (lugar donde el material a tratar es colocado) es cubierta totalmente por la región de la descarga luminiscente; donde el bombardeo de los iones y partículas neutras le transfiere energía suficiente para producir el calentamiento del cátodo [7].

En algunos trabajos se encuentra el estudio de los parámetros de la descarga luminiscente, entre los que se encuentran el campo eléctrico, el ancho de la zona de la caída catódica o región catódica, la temperatura del gas, la densidad de corriente y el flujo de calor hacia el cátodo $[8,9,10]$.

La temperatura del gas y la temperatura de la superficie del cátodo son dos parámetros cuyo modelo teórico y datos experimentales no son consistentes $[8,11]$, por lo cual se hace necesario realizar trabajos para contribuir a la comprensión de estos fenómenos y proponer aplicaciones basadas en estas características.

En este trabajo se propone el uso de la descarga luminiscente de baja presión como fuente de calor. Para esto, bajo ciertas condiciones de presión de la descarga, a través de un reactor cilíndrico diseñado especialmente se calentó una masa de agua y se comparó la potencia y energía consumidas con las presentes en un elemento calefactor convencional que emplea resistencias eléctricas.

\section{Metodología}

Se diseñó un reactor cilíndrico en acero al carbono, constituido por dos electrodos concéntricos, donde el electrodo interno constituye el cátodo de la descarga y el electrodo externo, que hace las veces de la cámara del reactor, constituye el ánodo como se ilustra en la figura 1.

El ánodo fue sellado en uno de los extremos mientras que el ánodo y el cátodo se acoplan entre sí a través de un material aislante (teflón). Las dimensiones del ánodo son: altura $20 \mathrm{~cm}$ y diámetro $4.5 \mathrm{~cm}$. $\mathrm{El}$ electrodo interior (cátodo) es de $18 \mathrm{~cm}$. de largo y $0.5 \mathrm{~cm}$. de diámetro. Para la atmósfera gaseosa de la descarga se utilizó argón a una presión de 2, 3, 4 y 5 Torr. El reactor se acopló a un recipiente calefactor comercial que funciona con base en resistencia eléctrica.

Como fuente de alimentación de la descarga se utilizó una fuente de corriente continua pulsada (obtenida por rectificación de onda completa de la señal alterna de la red). La descarga fue establecida a una corriente de $0.45 \mathrm{~A}$. a voltajes diferentes, dependientes de la presión. A presión de 2 Torr también se observó el comportamiento de la descarga en función de la corriente y de la atmosfera gaseosa.

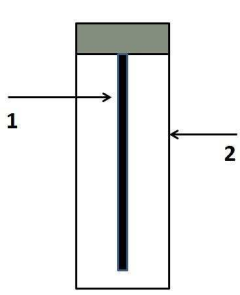

a)

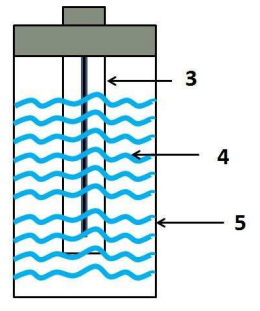

b)
Figura 1. a) Esquema del reactor empleado: 1) cátodo 2) ánodo. b); Esquema del montaje para transferir calor a través de la descarga luminiscente: 3 ) reactor 4) masa de agua 5) recipiente calefactor comercial.

Se realizaron medidas de corriente y voltaje para determinar la potencia y tiempo de funcionamiento, la energía consumida, necesaria para elevar la temperatura de $1.5 \mathrm{~L}$. de agua desde $20^{\circ} \mathrm{C}$. por debajo de $\operatorname{los} 40^{\circ} \mathrm{C}$., para evitar pérdida de energía por radiación. Estos mismos valores fueron medidos para el calefactor resistivo comercial. Con estos datos se 
estimó el calor cedido a la masa de agua y así se obtuvo la eficiencia de cada dispositivo.

\section{Resultados y discusión}

En la Tabla 1. se registran los datos de corriente $(I)$, voltaje $(V)$ y tiempo de funcionamiento del dispositivo resistivo comercial y el basado en la descarga luminiscente en función de la presión para una corriente fija de 0.45 Torr empleando gas de argón. Con éstos fue calculada la potencia:

$$
P=I V
$$

y el consumo de energía:

$$
C=P t
$$

A partir del cambio de temperatura (Ti: temperatura inicial y Tf: temperatura final) de la cantidad de agua utilizada ( $\mathrm{M}=1.5 \mathrm{~L}$.) se determina la cantidad de energía absorbida por el agua:

$$
Q=(c(M+k)(T i-T f))
$$

donde $c$ es la capacidad calorífica del agua y $k$ el equivalente en agua del calorímetro; la eficiencia fue calculada de la forma:

$$
e=(C-Q / C) * 100
$$

para cada uno de los sistemas.

En las Tablas 1,2, 3 se observa la disminución de la tensión de la descarga cuando se aumenta la presión de la atmósfera gaseosa entre 3 y 4 torr. Este comportamiento es debido a la mayor facilidad para ionizar el gas de la descarga cuando aumenta su concentración, consecuentemente, requiriéndose de menos tensión para generar las especies activas del plasma [12]. Este comportamiento se puede apreciar claramente en la disminución de la potencia con el aumento de la presión de la descarga, pues se requiere de menos potencia para mantener la misma corriente. No obstante existe un límite de presión para el cual la descarga puede mantenerse, pues la densidad del gas aumenta hasta el punto de disminuir considerablemente el recorrido libre medio de las partículas dificultándose la ionización del gas. Este comportamiento puede observarse a 2 y 5 Torr de presión, cuando la tensión de la descarga empieza a incrementarse. En la Tabla 1., también se observa una eficiencia térmica, en el reactor diseñado, com-

\begin{tabular}{|c|c|c|c|c|c|c|c|c|}
\hline & $\mathrm{I}(\mathrm{A})$ & $\mathrm{V}(\mathrm{V})$ & $\mathrm{P}$ (Torr) & $\mathrm{Ti}\left({ }^{\circ} \mathrm{C}\right)$ & $\operatorname{Tf}\left({ }^{\circ} \mathrm{C}\right)$ & $\mathrm{t}(\mathrm{s})$ & Consumo (J) & Eficiencia (\%) \\
\hline & \multicolumn{8}{|c|}{ Dispositivo comercial } \\
\hline & 8.90 & 127 & $1,130.30$ & 20 & 31.4 & 70.00 & $79,121.00$ & 92.77 \\
\hline Presión & Reactor & & & & & & & \\
\hline 2 & 0.45 & 386 & 173.70 & 20 & 30.0 & 375.65 & $65,250.41$ & 87.22 \\
\hline 3 & 0.45 & 411 & 184.95 & 20 & 25.5 & 191.05 & $35,334.70$ & 85.87 \\
\hline 4 & 0.45 & 401 & 180.45 & 20 & 26.5 & 240.67 & $43,428.90$ & 89.31 \\
\hline 5 & 0.45 & 427 & 183.61 & 20 & 27.0 & 249.78 & $45,864.00$ & 87.58 \\
\hline
\end{tabular}
parable a la del dispositivo comercial.

Tabla 1. Parámetros de funcionamiento para el dispositivo comercial y el reactor proyectado a varias presiones de la descarga en argón

Tabla 2. Comportamiento del reactor para varias corrientes en atmósfera de argón

\begin{tabular}{cccccccc}
\hline $\mathrm{I}(\mathrm{A})$ & $\mathrm{V}(\mathrm{V})$ & $\mathrm{P}($ Torr $)$ & $\mathrm{Ti}\left({ }^{\circ} \mathrm{C}\right)$ & $\mathrm{Tf}\left({ }^{\circ} \mathrm{C}\right)$ & $\mathrm{t}(\mathrm{s})$ & Consumo $(\mathrm{J})$ & Eficiencia $(\%)$ \\
\hline 0.26 & 376 & 97.76 & 20 & 28.5 & 569.86 & $55,709.51$ & 87.61 \\
0.45 & 386 & 173.70 & 20 & 30.0 & 375.65 & $65,250.41$ & 87.22 \\
0.55 & 390 & 214.50 & 20 & 30.0 & 319.90 & $68,618.55$ & 91.72 \\
0.62 & 396 & 245.52 & 20 & 30.0 & 264.66 & $64,979.32$ & 86.86 \\
\hline
\end{tabular}

Tabla 3. Comportamiento del reactor a 2 Torr en diferentes atmósferas gaseosas

\begin{tabular}{ccccccccc}
\hline $\mathrm{Gas}$ & $\mathrm{I}(\mathrm{A})$ & $\mathrm{V}(\mathrm{V})$ & $\mathrm{P}($ Torr $)$ & $\mathrm{Ti}\left({ }^{\circ} \mathrm{C}\right)$ & $\mathrm{Tf}\left({ }^{\circ} \mathrm{C}\right)$ & $\mathrm{t}(\mathrm{s})$ & Consumo $(\mathrm{J})$ & Eficiencia $(\%)$ \\
\hline $\mathrm{Ar}$ & 0.45 & 386 & 173.70 & 20 & 30.0 & 375.65 & $65,250.41$ & 87.22 \\
$\mathrm{~N}$ & 0.45 & 443 & 199.35 & 20 & 26.0 & 225.77 & $45,007.25$ & 92.65 \\
$\mathrm{H}$ & 0.50 & 406 & 203.00 & 20 & 24.0 & 149.51 & $30,350.53$ & 90.15 \\
\hline
\end{tabular}


En la Tabla 2., se registran los datos para el reactor funcionando en atmósfera de argón a 2 Torr de presión y diferentes corrientes. En la Tabla 3. se registra el comportamiento a 2 Torr de presión en diferentes atmósferas.

En los valores de corriente y voltaje de la descarga, de la Tabla 2, se observa un comportamiento similar al presentado en la región de la descarga luminiscencia anormal [12]. De forma similar a la Tabla 1, también se obtiene una eficiencia térmica, comparable a la del dispositivo comercial.

En la Tabla 3., se observa una diferencia del voltaje y, consecuentemente, de la potencia de la descarga, debido a la atmósfera gaseosa. La transferencia de energía cinética al cátodo y el calentamiento del mismo, se reducen por la disminución de la masa de las especies atómicas del gas. Esto se debe a la mayor dificultad para generar electrones secundarios del cátodo de la descarga con especies menos masivas [13].

\section{Referencias}

[1] K. N. Pandiyaraj, V. Selvarajan, R. R. Deshmukh, C. P. Yoganand, S. Balasubramanian, S. Maruthamuthu, "Low Pressure DC Glow Discharge Air Plasma Surface Treatment of Polyethylene (PE) Film for Improvement of Adhesive Properties", Plasma Sci Technol, vol. 15, pp. 56-63, 2013.

[2] J. L. Shohet, "Plasma-Aided Manufacturing", IEEE Trans. Plasma Sci, vol. 19, pp. 725-733, 1991.

[3] C.J. Scheuer, R.P. Cardoso, R. Pereira, M. Mafra y S.F. Brunatto, "Low temperature plasma carburizing of martensitic stainless Steel", Materials Science and Engineering A, vol. 539, pp. 369-372, 2012.

[4] A. Sarmiento Santos, J. Roa Rojas, D. Martínez, E. Vera, C.A. Parra Vargas y U. Fuentes Guerrero, "An Approach of the Sintering YBa2Cu3O7?? System ", Journal of Superconductivity and Novel Magnetism, vol. 26, pp. 2247-2251, 2013.

\section{Conclusiones}

Se diseño y puso en funcionamiento un reactor para aplicar la descarga luminiscente al calentamiento de líquidos con eficiencia comparable a la de los dispositivos resistivos comerciales. Dicho dispositivo funciona en el régimen de la descarga luminiscente anormal, reflejando su comportamiento característico en función de la presión y del tipo de gas a través del cual se genera la descarga. En términos de eficiencia, con atmósfera de nitrógeno a 2 Torr y 0.45 A se obtuvo el valor más alto.

Debido al interés en ahorrar energía con estos dispositivos, se ve la necesidad de realizar más estudios presentando la tecnología de la descarga luminiscente como un candidato potencial en el desarrollo de dispositivos para la trasferencia de calor en aplicaciones de la vida diaria y aplicaciones industriales que requieren el calentamiento para diversos fines. De esta forma se presenta una aplicación de la tecnología del plasma, no explorada con anterioridad según las fuentes consultadas.

[5] M. Belenguer, Ganciu, Guillot and T. Nelis, "Pulsed glow discharges for analytical applications ", Spectrochimica Acta Part B, vol. 64, pp. 623-641, 2009.

[6] X. Wanga, M. Zhoua y X. Jin, "Application of glow discharge plasma for wastewater treatment", Electrochimica Acta, vol. 83, pp. 501-512, 2012.

[7] S. F. Brunatto y N. V. Guimaraes, "Plasma assisted heat treatment: annealing", Journal of Physics. D: Applied. Physics, vol. 42, pp. 052003-052007, 2009.

[8] V. I. Arkhipenko, S. M. Zgirovski İ, A. A. Kirillov, y L. V. Simonchik, "Cathode Fall Parameters of a Self-Sustained Normal Glow Discharge in AtmosphericPressure Helium", Plasma Physics Reports, vol. 28, no. 10, pp. 858-865, 2002. Translated from Fizika Plazmy, Vol. 28, No. 10, pp. 930-938, 2002. 
[9] L.L. Alves, "Fluid modelling of the positive column of direct-current glow discharges", Plasma Sources Science Technology, vol. 16, pp. 557-569, 2007.

[10] V. I. Arkhipenko, A. A. Kirillov, Y. A. Safronau, L. V. Simonchik y S. M. Zgirouski, "Influence of cathode temperature on the parameters of an atmospheric pressure dc glow discharge", Plasma Sources Science Technology, vol. 17, pp. 045017-045027, 2008.
[11] G.G. Bondarenko, V.I. Kristya y M.I. Supelnyak, "Calculation of the electrode surface temperature in the normal glow discharge", Vacuum, vol. 86, pp. 854-856, 2012.

[12] B, Chapman, "Glow Discharge Processes", John Wiley and Sons, New York, 1980.

[13] A. von Engel, "Ionized Gases", JAmerican Institute of Physics, 1997. 
